\title{
Numerical modelling of nonlinear thermo-electro-elasticity
}

2017, Vol. 22(II) 2196-2213

(C) The Author(s) 2016

Reprints and permissions:

sagepub.co.uk/journalsPermissions.nav

DOI: $10.1177 / 1081286517729867$

journals.sagepub.com/home/mms

\author{
Markus Mehnert, Jean-Paul Pelteret and Paul Steinmann \\ Chair of Applied Mechanics, University of Erlangen-Nuremberg, Erlangen, Germany
}

Received 18 April 2017; accepted I4 August 2017

\begin{abstract}
This work presents the numerical modelling of nonlinear thermo-electro-elasticity in the context of electro-active polymers (EAPs). EAPs are characterised by their electro-mechanical coupling behaviour that converts electrical into mechanical energy. As polymeric materials in general are sensitive to the influence of temperature, thermal effects play an important role in the material behaviour of EAPs. Based on a thermo-electro-mechanically coupled constitutive framework presented in an earlier contribution, a variational formulation is developed and the finite-element method is employed to solve the nonlinear thermo-electro-mechanical coupling problem. The numerical implementation is studied by means of several examples.
\end{abstract}

\section{Keywords}

Nonlinear electro-elasticity, nonlinear thermo-elasticity, electro-active polymer, thermo-electro-mechanical coupling, finite-element method

\section{Dedication}

This contribution is dedicated to Gérard A Maugin whose work has been a source of both knowledge and inspiration. By pioneering theories and frameworks in the field of mathematical modelling of electro- and magneto-elasticity [1-3], he has helped lay the groundwork for the advances in the simulation of smart materials that we enjoy today. The books Continuum mechanics of electromagnetic solids [4] and Electrodynamics of continua I: Foundations and solid media [5] authored in cooperation with Eringen are especially brilliant summaries of the interplay of mechanics and electrodynamics within the context of continuum mechanics. Along with the modelling and simulation of electro-active materials, Gérard Maugin has also made important contributions to the study of their defects [6] through the application of material forces [7, 8].

\section{Introduction}

The popularity of electro-active polymers (EAPs) among the smart materials community has increased significantly within the past four decades. This particular class of material reacts to excitation by an electric field with significant displacements and a change in the material behaviour. As EAPs can generate larger deformations than traditional actuators like piezoelectrics, shape memory alloys or electro-magnetic motors, they have become popular in a wide range of applications such as artificial muscles, sensors, generators, microfluidic pumping systems and adaptable optics [9-15]. Their potentially low production cost, low weight and sufficiently short response time in combination with large deformation capabilities make them suitable for use in 
a broad range of industries $[10,16]$. EAPs can be split into two main categories based on the deformation mechanism [11, 17]: for ionic electro-active polymers (IEAPs), intramolecular forces are the main factor that leads to electrostriction. For electronic electro-active polymers (EEAPs) the Maxwell stress originating from electrostatic forces between electric charges instigates a mechanical response.

In the form of a thin film sandwiched between two compliant electrodes these EEAPs are used as actuators. When an electric potential difference is applied the thin film is squeezed in the direction of the resulting field. As the polymeric material is idealised as nearly incompressible [18], the material expands in the transverse planar direction. During the operation of EEAPs large temperature gradients may occur due to external thermal loading, large deformations or high electric loads needed for the activation.

In a number of recent publications the viscoelastic behaviour of EAPs has been modelled. For example, in $[19,20]$ Ask et al. used a phenomenological approach to model the electrostrictive behaviour of a polyurethane (PU) elastomer and validated their model using data from Johlitz et al. [21] and Diaconu et al. [22]. Later Büschel presented an electro-viscoelastic model in which the free energy is based on an Ogden-type energy function [23], whereas Saxena et al. [24, 25] developed a model in which the vector-valued electric field is decomposed additively into an equilibrium and a non-equilibrium part. In order to capture electromechanical instabilities Park et al. proposed a model that included inertial effects in which the mechanical and electrical governing equations were solved monolithically [26]. None of these constitutive models, however, takes into account the influence of the temperature on the material response. When we consider the possible application fields, such as adaptable valves in car engines or adjustable noise reduction systems in aeroplanes, it is clear that EAPs can be exposed to a large range of temperatures. Therefore it is crucial not only to analyse the electro-mechanical coupling effects but also to examine the influence of thermal loading in order to gain a better understanding of the parameters that control the EAP activation force and deformation.

Based on a preceding contribution [27] we focus this work on the numerical modelling of the nonlinear thermo-electro-elastic behaviour of a system made of these smart materials. The electro-thermo-mechanical system is partitioned into two subproblems using an isothermal split in which one subproblem is solved at a constant temperature distribution and the second, at a constant displacement and electric potential. These coupling schemes have been presented in a large number of papers, for example in [28-30]. The accuracy and the capabilities of the implementation are demonstrated by a number of numerical examples.

\section{Basics of nonlinear thermo-electro-mechanics}

\section{I. Kinematics}

We investigate a nonlinearly deforming body $\mathcal{B}$ with reference configuration $\mathcal{B}_{0}$ and spatial configuration $\mathcal{B}_{t}$. The position of a point in the reference configuration $\mathcal{B}_{0}$ is specified by the position vector $\boldsymbol{X}$. The corresponding position vector in the deformed configuration $\mathcal{B}_{t}$ is denoted $\boldsymbol{x}$. The nonlinear deformation map $\boldsymbol{\varphi}$ defines the connection between these two configurations such that $x=\varphi(X)$. Furthermore, the deformation gradient $F$ can be defined as the gradient of the deformation map with respect to the material coordinates. Its determinant $J$, the Jacobian determinant, must be positive in order to prevent unphysical deformation. As a stretch measure we introduce the right Cauchy-Green tensor $\boldsymbol{C}$. Collectively, the above are summarised as

$$
\boldsymbol{F}=\operatorname{Grad} \boldsymbol{\varphi} ; \quad J=\operatorname{det} \boldsymbol{F} ; \quad \boldsymbol{C}=\boldsymbol{F}^{\mathrm{T}} \boldsymbol{F} .
$$

\subsection{Balance laws in electrostatics}

In the following section the pertinent electro-mechanical balance equations are presented. These are well established in the works of Maugin [4], Voltairas et al. [31], Griffiths [32], and Dorfmann and Ogden [33], amongst others.

3.2.I. Spatial configuration. The electric displacement $d$ is computed by the constitutive relation

$$
\mathbb{d}=\varepsilon_{0} \mathbb{e}+\mathbb{P}=: \mathbb{d}^{\varepsilon}+\mathbb{P} \text { in } \mathcal{B}_{t},
$$

with the electric field $\mathbb{e}$, the vacuum electric permittivity $\varepsilon_{0}=8.85 \times 10^{-12} \mathrm{~F} / \mathrm{m}$ and the electric polarisation $P$. In vacuo the polarisation vanishes and the remaining term gives the vacuum electric displacement as $\mathbb{d}^{\varepsilon}=\varepsilon_{0} \mathbb{e}^{\text {. }}$ 
characterised The electric problem is characterised by Maxwell equations. In the absence of free currents and free electric charges it holds for the deformed body that

$$
\operatorname{curl} \mathbb{e}=\mathbf{0}, \quad \operatorname{div} \mathbb{d}=0 \quad \text { in } \mathcal{B}_{t},
$$

where curl and div denote the corresponding differential operators with respect to the position vectors $\boldsymbol{x}$ in $\mathcal{B}_{t}$. Equation (3) $)_{1}$ is satisfied when the electric field is derived from a scalar potential $\phi[34,35]$; thus $\bullet$ is defined as the gradient of the electric potential with respect to the spatial coordinates such that

$$
\mathbb{e}=-\operatorname{grad} \phi
$$

Including mechanical body forces $\boldsymbol{b}_{t}$ the equilibrium equation in nonlinear electro-mechanics can be expressed as

$$
\operatorname{div} \boldsymbol{\sigma}+\boldsymbol{b}_{t}^{\text {pon }}+\boldsymbol{b}_{t}:=\operatorname{div} \boldsymbol{\sigma}^{\text {tot }}+\boldsymbol{b}_{t}=\mathbf{0} \quad \text { in } \mathcal{B}_{t} .
$$

The total Cauchy-type symmetric stress tensor $\sigma^{\text {tot }}[33]$ can be additively split into a non-symmetric mechanical Cauchy stress $\sigma$ and the ponderomotive stress $\sigma^{\text {pon }}[36]$ satisfying

$$
\operatorname{div} \boldsymbol{\sigma}^{\text {pon }}:=\boldsymbol{b}_{t}^{\text {pon }}=\operatorname{grad} \mathbb{e} \cdot \mathbb{P}
$$

The ponderomotive stress is composed of the symmetric Maxwell stress $\sigma^{\max }$ and a non-symmetric polarisation stress $\sigma^{\mathrm{pol}}[37,38]$ which are

$$
\boldsymbol{\sigma}^{\max }=-\frac{1}{2} \varepsilon_{0}[\mathbb{e} \cdot \mathbb{e}] \boldsymbol{i}+\varepsilon_{0} \mathbb{e} \otimes \mathbb{e}, \quad \boldsymbol{\sigma}^{\mathrm{pol}}=\mathbb{e} \otimes \mathbb{P},
$$

with $i$ representing the second-order spatial identity tensor.

At the boundary of the body $\partial \mathcal{B}_{t}$ and any internal surface of discontinuity, the total stress tensor must satisfy the jump condition

$$
-\llbracket \boldsymbol{\sigma}^{\mathrm{tot}} \rrbracket \cdot \boldsymbol{n}=\boldsymbol{t}_{t}^{\mathrm{p}} \quad \text { on } \partial \mathcal{B}_{t},
$$

where $\boldsymbol{t}_{t}^{\mathrm{p}}$ are the imposed mechanical tractions and $\boldsymbol{n}$ is the surface normal on $\partial \mathcal{B}_{t}$ pointing outwards. Here $\llbracket \bullet \rrbracket=[\bullet]^{\text {out }}-[\bullet]^{\text {in }}$ implies the jump of a quantity at the interface between the bulk material and the free space surrounding the body. When the influence of the free space is neglected this jump condition simplifies to the standard boundary condition. The boundary $\partial \mathcal{B}_{t}$ is split into a part $\partial \mathcal{B}_{t}^{\varphi}$ on which Dirichlet boundary conditions for the deformation map $\varphi$ are prescribed, and $\partial \mathcal{B}_{t}^{\mathrm{t}}$, where mechanical tractions $t_{t}^{\mathrm{p}}$ are imposed such that

$$
\boldsymbol{\varphi}=\boldsymbol{\varphi}^{\mathrm{p}} \quad \text { on } \partial \mathcal{B}_{t}^{\varphi} \quad \text { and } \quad \boldsymbol{\sigma}^{\mathrm{tot}} \cdot \boldsymbol{n}=\boldsymbol{t}_{t}^{\mathrm{p}} \quad \text { on } \partial \mathcal{B}_{t}^{\mathrm{t}} .
$$

Similarly the jump conditions for the spatial electric field vector $\mathbb{e}$, as well as the electric displacement $\mathbb{d}$ at the boundary and on possible discontinuity surfaces of the body, are

$$
\llbracket \mathbb{e} \times \boldsymbol{n}=\mathbf{0} \quad \text { and } \quad \llbracket \llbracket \rrbracket \cdot \boldsymbol{n}=\hat{\varrho}_{t}^{f} \quad \text { on } \partial \mathcal{B}_{t},
$$

where $\hat{\varrho}_{t}^{f}$ is defined as the density of free surface charges per deformed area [37]. As for the mechanical quantities the jump conditions reduce to classical boundary conditions when not considering the influence of the surrounding free space. In combination with (4) the boundary $\partial \mathcal{B}_{t}$ is split into a part $\partial \mathcal{B}_{t}^{\phi}$ on which Dirichlet boundary conditions for the electric potential are prescribed and $\partial \mathcal{B}_{t}^{\varrho}$ where Neumann conditions

$$
\phi=\phi^{\mathrm{p}} \quad \text { on } \partial \mathcal{B}_{t}^{\phi} \quad \text { and } \quad-\mathbb{d} \cdot \boldsymbol{n}=\hat{\varrho}_{t}^{f} \quad \text { on } \partial \mathcal{B}_{t}^{\varrho}
$$

have to be satisfied. 
3.2.2. Material configuration. Various quantities described in the previous section are now transformed from the spatial into the material configuration. For the electric field, the electric displacement and the polarisation the result of this transformation is

$$
\mathbb{E}=\boldsymbol{F}^{\mathrm{T}} \cdot \mathbb{e}, \quad \mathbb{D}=J \boldsymbol{F}^{-1} \cdot \mathbb{d}, \quad \mathbb{P}=J \boldsymbol{F}^{-1} \cdot \mathbb{P},
$$

which leads to the Maxwell equations in the form

$$
\operatorname{Curl} \mathbb{E}=\mathbf{0}, \quad \operatorname{Div} \mathbb{D}=0 .
$$

In the material configuration the differential operators Curl and Div are defined with respect to the material position vector $\boldsymbol{X}$. Again the first equation is satisfied when the electric field is derived from a scalar potential such that

$$
\mathbb{E}=-\operatorname{Grad} \phi \quad \text { in } \mathcal{B}_{0} .
$$

As presented in [35] the electric displacement can be defined as

$$
\mathbb{D}=\varepsilon_{0} J \boldsymbol{C}^{-1} \cdot \mathbb{E}+\mathbb{P}=: \mathbb{D}^{\varepsilon}+\mathbb{P} \text { in } \mathcal{B}_{0},
$$

with the vacuum electric displacement $\mathbb{D}^{\varepsilon}:=\varepsilon_{0} J \boldsymbol{C}^{-1} \cdot \mathbb{E}$. This formulation follows from a pull-back of the spatial format given in equation (2). The pull-back of the total Cauchy stress $\sigma^{\text {tot }}$ is the total Piola stress tensor

$$
\boldsymbol{P}^{\mathrm{tot}}=J \boldsymbol{\sigma}^{\mathrm{tot}} \cdot \boldsymbol{F}^{-\mathrm{T}}
$$

which is composed of a mechanical part $\boldsymbol{P}$ and a ponderomotive part $\boldsymbol{P}^{\text {pon }}$, with

$$
\boldsymbol{P}^{\mathrm{tot}}=\boldsymbol{P}+\boldsymbol{P}^{\mathrm{pon}}=\boldsymbol{P}+\boldsymbol{P}^{\mathrm{max}}+\boldsymbol{P}^{\mathrm{pol}},
$$

whereby $\boldsymbol{P}^{\text {pon }}$ decomposes into the polarisation stress $\boldsymbol{P}^{\text {pol }}$ and the Maxwell stress $\boldsymbol{P}^{\text {max }}$ through the relations

$$
\boldsymbol{P}^{\mathrm{pol}}=\mathbb{e} \otimes \mathbb{P}, \boldsymbol{P}^{\mathrm{max}}=-\frac{1}{2} \varepsilon_{0} J \boldsymbol{C}^{-1}:[\mathbb{E} \otimes \mathbb{E}] \boldsymbol{F}^{-\mathrm{T}}+\mathbb{e} \otimes \mathbb{D}^{\varepsilon} .
$$

In the undeformed configuration the balance of linear momentum (5) is defined as

$$
\operatorname{Div} \boldsymbol{P}^{\mathrm{tot}}+\boldsymbol{b}_{0}=\mathbf{0}
$$

and the jump conditions are translated to the material configuration such that

$$
\llbracket \mathbb{D} \rrbracket \cdot N=\hat{\varrho}_{0}^{f}, \quad \text { and } \quad-\llbracket \boldsymbol{P}^{\mathrm{tot}} \rrbracket \cdot \boldsymbol{N}=\boldsymbol{t}_{0}^{p} \quad \text { on } \partial \mathcal{B}_{0} .
$$

This results in the case that the free space is not considered in the boundary conditions

$$
\begin{array}{ll}
-\mathbb{D} \cdot \boldsymbol{N}=\hat{\varrho}_{0}^{f} & \text { on } \partial \mathcal{B}_{0}^{\varrho}, \\
\boldsymbol{P}^{\text {tot }} \cdot \boldsymbol{N}=\boldsymbol{t}_{0}^{p} & \text { on } \partial \mathcal{B}_{0}^{t} .
\end{array}
$$

\subsection{Heat equation}

In this chapter basic relations in thermo-electro-elasticity are presented. A more detailed description of the basics of these equations and their derivations can be found in the works of, for example, Erbts et al. [30], Holzapfel [39], Miehe [40] and Vogel [35].

We start by introducing the first law of thermodynamics as the balance between the change in the energy $\mathcal{E}$ and the sum of external mechanical power $\mathcal{P}_{\text {ext }}$ and the non-mechanical power $\mathcal{Q}$, which contains both the thermal power $\mathcal{Q}^{\text {thm }}$ and ponderomotive power density $\mathcal{Q}^{\text {pon }}$ :

$$
\dot{\mathcal{E}}=\dot{\mathcal{U}}+\dot{\mathcal{K}}=\mathcal{P}_{\text {ext }}+\mathcal{Q}=\mathcal{P}_{\text {ext }}+\mathcal{Q}^{\text {thm }}+\mathcal{Q}^{\text {pon }} .
$$


Here the energy is decomposed into the internal energy $\mathcal{U}$ and the kinetic energy $\mathcal{K}$ (that, however, vanishes in the quasi-static case). As demonstrated in [35] from this we can derive the local form of the balance of energy in the material configuration as

$$
\dot{\mathcal{U}}=\boldsymbol{P}: \dot{\boldsymbol{F}}-\operatorname{Div} \boldsymbol{Q}+\mathcal{R}+\mathbb{E} \cdot \dot{\mathbb{P}}+\boldsymbol{P}^{\mathrm{pol}}: \dot{\boldsymbol{F}},
$$

where $\dot{\mathcal{U}}$ is the change in the internal energy density per unit undeformed volume, $\mathcal{R}$ are the heat sources and $\boldsymbol{Q}$ defines the heat flux vector in the material configuration. It is connected to the absolute temperature $\Theta$ by the definition $\boldsymbol{Q}:=-\kappa J \boldsymbol{C}^{-1}$. Grad $\Theta$ where $\kappa$ is the isotropic heat conductivity and the negative sign expresses the physical observation that heat flows in the direction of a negative temperature gradient. When we introduce the entropy $H$ we can formulate the localisation of the Clausius-Duhem inequality as

$$
\mathcal{D}=\Theta \dot{H}-\frac{\boldsymbol{Q}}{\Theta} \cdot \operatorname{grad} \Theta-\dot{\mathcal{U}}+\boldsymbol{P}: \dot{\boldsymbol{F}}+\mathbb{E} \cdot \dot{\mathbb{P}}+\boldsymbol{P}^{\mathrm{pol}}: \dot{\boldsymbol{F}} \geq 0,
$$

which contains the dissipation power density $\mathcal{D}=\mathcal{D}(\boldsymbol{X}, t) \geq 0$. As shown in [41] this term can be split into a dissipation power density due to heat conduction $\mathcal{D}^{\text {con }}=-\frac{\boldsymbol{Q}}{\Theta} \cdot \operatorname{grad} \Theta \geq 0$ and a local dissipation power density $\mathcal{D}^{\text {loc }}$ :

$$
\mathcal{D}^{\text {loc }}=\Theta \dot{H}-\dot{\mathcal{U}}+\boldsymbol{P}: \dot{\boldsymbol{F}}+\mathbb{E} \cdot \dot{\mathbb{P}}+\boldsymbol{P}^{\mathrm{pol}}: \dot{\boldsymbol{F}} \geq 0,
$$

which is the Clausius-Planck inequality. For a reversible process the dissipation is equal to zero whereas the inequality holds for any irreversible process. Using a Legendre transformation [42] of the form

$$
\Psi(\boldsymbol{F}, \Theta, \mathbb{E})=\mathcal{U}-\Theta H-\mathbb{E} \cdot \mathbb{P},
$$

we introduce the free energy density $\Psi(\boldsymbol{F}, \theta, \mathbb{E})$ that depends on the current state of deformation, the electric field and the temperature. With this we can reformulate the Clausius-Planck inequality as

$$
\mathcal{D}^{\text {loc }}=-\dot{\Psi}-\dot{\Theta} H+\left[\boldsymbol{P}+\boldsymbol{P}^{\mathrm{pol}}\right]: \dot{\boldsymbol{F}}-\dot{\mathbb{E}} \cdot \mathbb{P} \geq 0 .
$$

Following $[43,44]$ we amend the free energy density $\Psi(\boldsymbol{F}, \theta, \mathbb{E})$ with a term $E(\boldsymbol{F}, \mathbb{E})$ representing the energy stored in the electric field. This leads to the augmented total free energy density

$$
\Omega(\boldsymbol{F}, \theta, \mathbb{E})=\Psi(\boldsymbol{F}, \theta, \mathbb{E})+E(\boldsymbol{F}, \mathbb{E})
$$

per unit volume in $\mathcal{B}_{0}$, which eventually renders a formulation of the Clausius-Duhem inequality containing the total Piola stress,

$$
\mathcal{D}^{\text {loc }}=-\dot{\Omega}-\dot{\Theta} H+\boldsymbol{P}^{\text {tot }}: \dot{\boldsymbol{F}}-\dot{\mathbb{E}} \cdot \mathbb{D} \geq 0 .
$$

As presented in [27] by means of the total free energy function, the constitutive relations for the total Piola stress, including the Maxwell stress, the electric displacement and the entropy can be computed by

$$
\boldsymbol{P}^{\mathrm{tot}}=\frac{\partial \Omega}{\partial \boldsymbol{F}}, \quad \text { with } \quad \boldsymbol{P}^{\max }=\frac{\partial E}{\partial \boldsymbol{F}}, \quad \mathbb{D}=-\frac{\partial \Omega}{\partial \mathbb{E}}, \quad H=-\frac{\partial \Omega}{\partial \theta} .
$$

Combining equations (23) and (27) we can reformulate the first law of thermodynamics in entropy form as

$$
\theta \dot{H}=\mathcal{R}-\operatorname{Div} \boldsymbol{Q}+\mathcal{D}^{\text {loc }} .
$$

Using the chain rule as in [27] we can expand the left-hand-side term of (31) to

$$
\Theta \dot{H}=-\Theta \frac{\partial^{2} \Omega}{\partial \Theta \partial \Theta} \dot{\Theta}-\Theta \frac{\partial^{2} \Omega}{\partial \boldsymbol{F} \partial \Theta}: \dot{\boldsymbol{F}}-\Theta \frac{\partial^{2} \Omega}{\partial \mathbb{E} \partial \Theta} \cdot \dot{\mathbb{E}} .
$$

With the constitutive relation of the entropy $H$ and the specific heat capacity $c_{0}=-\Theta \frac{\partial^{2} \Omega}{\partial \Theta^{2}}$ equation (31) is reformulated into the heat conduction equation in the form

$$
c_{0} \dot{\theta}=\mathcal{R}-\operatorname{Div} \boldsymbol{Q}+\underbrace{\theta \partial_{\theta}\left[\boldsymbol{P}^{\mathrm{tot}}: \dot{\boldsymbol{F}}-\mathbb{D} \cdot \dot{\mathbb{E}}\right]}_{\mathcal{H}}+\mathcal{D}^{\mathrm{loc}} .
$$


Here the term $\mathcal{H}$ denotes the structural thermo-mechanical and thermo-electric heating/cooling effect, the former of which is also known as the Gough-Joule effect. As we are not considering local dissipation effects the term $\mathcal{D}^{\text {loc }}$ vanishes in (33), thus giving $\mathcal{D}^{\text {loc }}=0$. Along the boundary $\partial \mathcal{B}_{0}=\partial \mathcal{B}_{0}^{\Theta} \cup \partial \mathcal{B}_{0}^{q}$ Dirichlet boundary conditions for the temperature and Neumann boundary conditions for the heat flux

$$
\Theta=\Theta^{p} \quad \text { on } \partial \mathcal{B}_{0}^{\Theta} \quad \text { and } \quad \boldsymbol{Q} \cdot \boldsymbol{N}=\bar{Q} \quad \text { on } \partial \mathcal{B}_{0}^{q}
$$

are imposed.

\section{Variational formulation}

The boundary value problem governed by equations (13), (19) and (33) in conjunction with the boundary conditions expressed in equations (21) and (34) can be solved with the finite-element method. To achieve this we have to reformulate this system of equations from the strong form into the weak form. Therefore all equations are multiplied by the variation of the respective unknown variable. The terms are integrated by parts and application of the divergence theorem provides the Neumann boundary conditions. This leads to the variational form of the coupled system, also known as the virtual work consisting of internal $(\bullet)_{\text {int }}$ and external $(\bullet)_{\text {ext }}$ contributions. For the electro-mechanical system $\mathcal{G}_{\varphi, \phi}$ with a fixed temperature field, as presented in $[34,35]$ for instance, these contributions read

$$
\begin{aligned}
\left.\mathcal{G}_{\boldsymbol{\varphi}, \phi}\left(\boldsymbol{\varphi}, \phi, \delta \boldsymbol{\varphi}, \delta \phi, \boldsymbol{t}_{0}^{p}, \boldsymbol{b}_{0}, \hat{\varrho}_{0}^{f}\right)\right|_{\Theta}=\underbrace{\int_{\mathcal{B}_{0}} \boldsymbol{P}^{\mathrm{tot}}: \nabla_{\boldsymbol{X}} \delta \boldsymbol{\varphi}+\mathbb{D} \cdot \nabla_{\boldsymbol{X}} \delta \phi \mathrm{d} V}_{\mathcal{G}_{\varphi, \phi}^{\text {int }}} \\
\underbrace{-\int_{\partial \mathcal{B}_{0}^{\mathrm{t}}} \boldsymbol{t}_{0}^{p} \cdot \delta \boldsymbol{\varphi} \mathrm{d} A-\int_{\mathcal{B}_{0}} \boldsymbol{b}_{0} \cdot \delta \boldsymbol{\varphi} \mathrm{d} V+\int_{\partial \mathcal{B}_{0}^{\varrho}} \hat{\varrho}_{0}^{f} \delta \phi \mathrm{d} A}_{\mathcal{G}_{\varphi, \phi}^{\text {ext }}}=0 .
\end{aligned}
$$

Similarly these terms can be derived for the thermal system at constant deformation and electric scalar potential as shown in $[29,30]$, which leads to

$$
\begin{aligned}
\left.\mathcal{G}_{\Theta}(\Theta, \delta \Theta, \mathcal{H}, R, \overline{\boldsymbol{Q}})\right|_{\varphi, \phi} & =\underbrace{\int_{\mathcal{B}_{0}} \rho_{0} c_{p} \dot{\Theta} \delta \Theta+\nabla_{\boldsymbol{X}} \delta \Theta \cdot \kappa \nabla_{\boldsymbol{X}} \Theta \mathrm{d} V}_{\mathcal{G}_{\Theta}^{\text {int }}} \\
& \underbrace{-\int_{\mathcal{B}_{0}}[\mathcal{H}+R] \delta \Theta \mathrm{d} V-\int_{\partial \mathcal{B}_{0}^{\Theta}} \overline{\boldsymbol{Q}} \delta \Theta \mathrm{d} A}_{\mathcal{G}_{\Theta}^{\text {ext }}}=0 .
\end{aligned}
$$

Now we will derive the linearisation of the equations (35) and (36) for the internal contributions of the virtual work $\mathcal{G}_{\Theta}^{\text {int }}$ and $\mathcal{G}_{\varphi, \phi}^{\text {int }}$.

The temperature rate is discretised by the first-order accurate implicit backward Euler scheme $\dot{\Theta} \approx\left[\Theta_{t}-\right.$ $\left.\Theta_{t-1}\right] / \Delta t$ where $t$ is the current time iterate and $\Delta t$ is the time increment. These nonlinear equations are then linearised, which leads to coupled terms in the mechanical and the electrical field. For temperature-independent or only weakly coupled material parameters it follows that

$$
\begin{aligned}
& \left.\Delta \mathcal{G}_{\boldsymbol{\varphi}, \phi}(\boldsymbol{\varphi}, \phi, \delta \boldsymbol{\varphi}, \delta \phi)\right|_{\Theta} \\
& =\int_{\mathcal{B}_{0}} \nabla_{\boldsymbol{X}} \delta \boldsymbol{\varphi}: \frac{\partial \boldsymbol{P}^{\mathrm{tot}}}{\partial \boldsymbol{F}}: \nabla_{\boldsymbol{X}} \Delta \boldsymbol{\varphi}-\nabla_{\boldsymbol{X}} \delta \boldsymbol{\varphi}: \frac{\partial \boldsymbol{P}^{\mathrm{tot}}}{\partial E} \cdot \nabla_{\boldsymbol{X}} \Delta \phi \mathrm{d} V \\
& \quad+\int_{\mathcal{B}_{0}} \nabla_{\boldsymbol{X}} \delta \phi \cdot \frac{\partial \mathbb{D}}{\partial \boldsymbol{F}}: \nabla_{\boldsymbol{X}} \Delta \boldsymbol{\varphi}-\nabla_{\boldsymbol{X}} \delta \phi \cdot \frac{\partial \mathbb{D}}{\partial E} \cdot \nabla_{\boldsymbol{X}} \Delta \phi \mathrm{d} V
\end{aligned}
$$

while the corresponding linearisation of the temperature variation is

$$
\left.\Delta \mathcal{G}_{\Theta}(\Theta, \delta \Theta)\right|_{\varphi, \phi}=\int_{\mathcal{B}_{0}} \frac{\rho_{0}}{\Delta t} c_{p} \delta \Theta \Delta \Theta+\nabla_{\boldsymbol{X}} \delta \Theta \cdot \kappa \nabla_{\boldsymbol{X}} \Delta \Theta \mathrm{d} V
$$


For a finite-element formulation the geometry of the body $\mathcal{B}_{0}$ has to be discretised. The respective field variables are approximated using the vector- and scalar-valued shape functions $N_{\alpha}$ and $N_{\alpha}$ corresponding to each degree of freedom $\alpha$. For a single element this leads to the following formulation for the displacement and its variation and their corresponding gradients:

$$
\begin{gathered}
\varphi(X) \approx \sum_{\alpha} \varphi_{\alpha} N_{\alpha}(X), \quad \delta \varphi(X) \approx \sum_{\alpha} \delta \varphi_{\alpha} N_{\alpha}(X) \\
\nabla_{X} \varphi(X) \approx \sum_{\alpha} \varphi_{\alpha} \frac{\partial \boldsymbol{N}_{\alpha}(\boldsymbol{X})}{\partial \boldsymbol{X}}, \quad \nabla_{\boldsymbol{X}} \delta \varphi(\boldsymbol{X}) \approx \sum_{\alpha} \delta \varphi_{\alpha} \frac{\partial \boldsymbol{N}_{\alpha}(\boldsymbol{X})}{\partial \boldsymbol{X}}
\end{gathered}
$$

The interpolation of the electric potential $\phi$ follows in a similar fashion, with

$$
\begin{gathered}
\phi(\boldsymbol{X}) \approx \sum_{\alpha} \phi_{\alpha} N_{\alpha}(\boldsymbol{X}), \quad \delta \phi(\boldsymbol{X}) \approx \sum_{\alpha} \delta \phi_{\alpha} N_{\alpha}, \\
\nabla_{\boldsymbol{X}} \phi(\boldsymbol{X}) \approx \sum_{\alpha} \phi_{\alpha} \frac{\partial N_{\alpha}}{\partial \boldsymbol{X}}, \quad \nabla_{\boldsymbol{X}} \delta \phi(\boldsymbol{X}) \approx \sum_{\alpha} \delta \phi_{\alpha} \frac{\partial N_{\alpha}}{\partial \boldsymbol{X}} .
\end{gathered}
$$

Finally the discretisation for the temperature reads

$$
\begin{gathered}
\Theta(\boldsymbol{X}) \approx \sum_{\alpha} \Theta_{\alpha} N_{\alpha}(\boldsymbol{X}), \quad \delta \Theta(\boldsymbol{X}) \approx \sum_{\alpha} \delta \Theta_{\alpha} N_{\alpha}, \\
\nabla_{\boldsymbol{X}} \Theta(\boldsymbol{X}) \approx \sum_{\alpha} \Theta_{\alpha} \frac{\partial N_{\alpha}}{\partial \boldsymbol{X}}, \quad \nabla_{\boldsymbol{X}} \delta \Theta(\boldsymbol{X}) \approx \sum_{\alpha} \delta \Theta_{\alpha} \frac{\partial N_{\alpha}}{\partial \boldsymbol{X}} .
\end{gathered}
$$

The finite-element formulation is completed by inserting the approximations into the respective functionals. For the residual equations (35), (36) this leads to

$$
\begin{aligned}
& \mathcal{G}_{u, v}\left(\boldsymbol{\varphi}, \varphi, \delta \boldsymbol{\varphi}, \delta \varphi, \boldsymbol{t}_{0}^{p}, \boldsymbol{b}_{0}, \hat{\varrho}_{0}^{f}\right)=\delta \varphi_{\alpha}\left[\int_{\mathcal{B}_{0}} \boldsymbol{P}^{\mathrm{tot}}: \frac{\partial \boldsymbol{N}_{\alpha}(\boldsymbol{X})}{\partial \boldsymbol{X}}\right] \\
&-\delta \varphi_{\alpha}\left[\int_{\partial \mathcal{B}_{0}^{\mathrm{t}}} \boldsymbol{t}_{0}^{p} \cdot \boldsymbol{N}_{\alpha}(\boldsymbol{X}) \mathrm{d} A-\int_{\mathcal{B}_{0}} \boldsymbol{b}_{0} \cdot \boldsymbol{N}_{\alpha}(\boldsymbol{X}) \mathrm{d} V\right] \\
&+\delta \phi_{\alpha}\left[\int_{\mathcal{B}_{0}} \mathbb{D} \cdot \frac{\partial \boldsymbol{N}_{\alpha}(\boldsymbol{X})}{\partial \boldsymbol{X}} \mathrm{d} V\right]+\delta \phi_{\alpha}\left[\int_{\partial \mathcal{B}_{0}^{\rho}} \hat{\varrho}_{0}^{f} \boldsymbol{N}_{\alpha}(\boldsymbol{X}) \mathrm{d} A\right]=0, \\
& \mathcal{G}_{\Theta}(\Theta, \delta \Theta, \mathcal{H}, R, \overline{\boldsymbol{Q}})= \delta \Theta_{\alpha}\left[\int_{\mathcal{B}_{0}} \rho_{0} c_{p} \dot{\Theta} N_{\alpha}+\frac{\partial N_{\alpha}}{\partial \boldsymbol{X}} \cdot \kappa \cdot \Theta_{\alpha} \frac{\partial N_{\alpha}}{\partial \boldsymbol{X}} \mathrm{d} V\right. \\
&\left.-\int_{\mathcal{B}_{0}}[\mathcal{H}+R] N_{\alpha} \mathrm{d} V-\int_{\partial \mathcal{B}_{0}^{\Theta}} \overline{\boldsymbol{Q}} N_{\alpha} \mathrm{d} A\right]=0 .
\end{aligned}
$$

\section{I. Partitioned formulation}

In this section a coupling scheme between the electro-mechanical system at a fixed temperature field and the thermal system at fixed deformation and electric potential is formulated, a method that is often termed the 'isothermal staggered approach'. For weakly coupled multi-field problems this approach has been applied frequently $[29,30,45]$. In contrast to this partitioned scheme, the entire system can also be solved with a monolithic approach for which the simultaneous solution of all field variables is computed. Therein the coupling between the different fields results in additional off-diagonal entries in the Jacobian matrix, and a reformulation of (37) and (38) is required.

In a partitioned approach, the computation of these off-diagonal entries containing cross-derivatives is avoided as each subproblem is considered individually. In the current case a mixture of both approaches is adopted, as the electro-mechanical problem is solved monolithically but the thermal problem is considered as a 
decoupled field and is connected to the rest of the system through the staggered transfer of solution fields. Therefore, the thermal problem is solved initially at fixed electric potential and displacement and subsequently the electro-mechanical problem is solved for a constant thermal field. Both subproblems are solved using Newton's method, wherein there is an information exchange after each iteration $i$.

The electro-mechanical system contains the stiffness sub-matrix $\boldsymbol{K}_{\varphi, \phi}$ consisting of a mechanical part $\boldsymbol{K}_{\varphi, \phi}^{\varphi \varphi}$, an electric part $K_{\varphi, \phi}^{\phi \phi}$ and the two mixed parts $\boldsymbol{K}_{\varphi, \phi}^{\varphi \phi}$ and $\boldsymbol{K}_{\varphi, \phi}^{\phi \varphi}$, which leads to

$$
\begin{aligned}
\boldsymbol{K}_{\boldsymbol{\varphi}, \phi} \cdot[\Delta \boldsymbol{\varphi}, \Delta \phi]=-\left.\mathcal{G}_{\varphi, \phi}\left(\boldsymbol{\varphi}^{(i)}, \phi^{(i)}\right)\right|_{\Theta} & \rightarrow[\boldsymbol{\varphi}, \phi]^{(i+1)} \\
=[\boldsymbol{\varphi}, \phi]^{(i)}+[\Delta \boldsymbol{\varphi}, \Delta \phi] & \text { with } \quad K_{\varphi, \phi}=\left[\begin{array}{ll}
\boldsymbol{K}_{\varphi, \phi}^{\varphi \varphi} & \boldsymbol{K}_{\varphi, \phi}^{\varphi \phi} \\
\boldsymbol{K}_{\varphi, \phi}^{\phi \varphi} & K_{\varphi, \phi}^{\phi \phi}
\end{array}\right] .
\end{aligned}
$$

The thermal problem with the stiffness sub-matrix $K_{\Theta}$ can be formulated as

$$
\begin{aligned}
& K_{\Theta} \Delta \Theta^{(i)}=-\left.\mathcal{G}_{\Theta}\left(\Theta^{(i)}\right)\right|_{\varphi, \varphi} \rightarrow \Theta^{(i+1)}=\Theta^{(i)}+\Delta \Theta^{(i)} \\
& \text { with } K_{\Theta}=\frac{\partial \mathcal{G}_{\Theta}}{\partial \Theta} .
\end{aligned}
$$

In other words, the response of one system is computed with a dependence on the second system so that the respective iteration residuals

$$
\boldsymbol{R}_{\varphi, \phi}^{(i)}=[\boldsymbol{\varphi}, \phi]^{(i+1)}-[\varphi, \phi]^{(i)} \text { and } \quad \boldsymbol{R}_{\Theta}^{(i)}=\Theta^{(i+1)}-\Theta^{(i)}
$$

vanish.

\section{Numerical examples}

In this section three nonlinear boundary value problems are investigated in order to validate the numerical implementation and furthermore to present its capabilities. These examples are evaluated using an in-house finite-element code developed using the finite-element (FE) library deal.II [46, 47] following the previously described total Lagrangian approach. Additionally, we use the software package Trilinos [48, 49] and p4est [50] to partition the grid. The electric potential $\phi$, the displacement $\varphi$ and the temperature $\Theta$ are discretised using linear shape functions. The resulting linear set of equations is solved using a direct solver. Due to the capacitor-like geometry of these problems, any contributions from the surrounding free space is negligible [51] and therefore ignored for the sake of simplicity.

The first example is the inflation and extension of a cylindrical tube. As the simple problem setup allows for an analytical solution, this example is used to demonstrate the validity of the finite-element implementation. The problem is subsequently extended by including thermo-electric and thermo-mechanical heating terms. As a second academic example a three-dimensional plate with a hole in its centre is subjected to electric loading and we illustrate the influence of the ramp-up time of the electric field by analysing both the deformation and the resulting temperature field. In the third and final example we present an idealised microfluidic pumping device employing electro-active material in the form of a diaphragm actuator. We investigate the influence of the fluid temperature on the functionality of the pump.

In all of the examples we consider a thermo-electro-mechanically coupled energy function in a form as derived in [27], namely

$$
\Psi(\boldsymbol{F}, \theta, \mathbb{E})=\frac{\theta}{\theta_{0}} W(\boldsymbol{F}, \mathbb{E})+c_{0}\left[\theta-\theta_{0}-\theta \ln \left(\frac{\theta}{\theta_{0}}\right)\right]-\left[\theta-\theta_{0}\right] M(\boldsymbol{F}) .
$$

The isothermal contribution $W(\boldsymbol{F}, \mathbb{E})$ is motivated by a neo-Hookean-type model of rubber-like materials given by

$$
W(\boldsymbol{F}, \mathbb{E})=\frac{\mu\left(I_{4}\right)}{2}\left[I_{1}-3\right]+c_{1} I_{4}+c_{2} I_{5} .
$$

Here the invariants $[5,39,52]$ are defined as $I_{1}=\operatorname{tr}(\boldsymbol{C}), I_{4}=[\mathbb{E} \otimes \mathbb{E}]: \boldsymbol{I}$ and $I_{5}=[\mathbb{E} \otimes \mathbb{E}]: \boldsymbol{C}$. The purely mechanical part of the coupled contribution is chosen as $M(\boldsymbol{F})=3 \kappa \alpha \ln (J)$ with the thermal expansion 


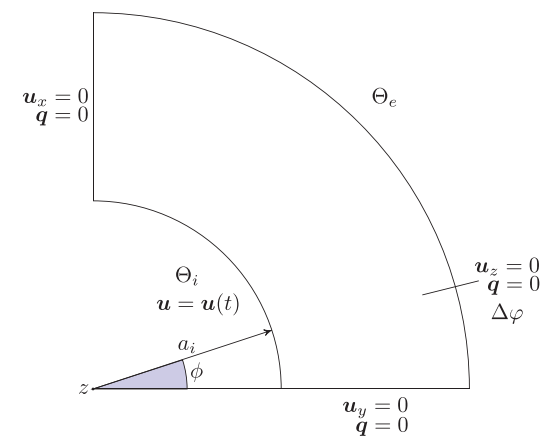

(a) Geometrical setup of the thick walled cylinder in the deformed configuration

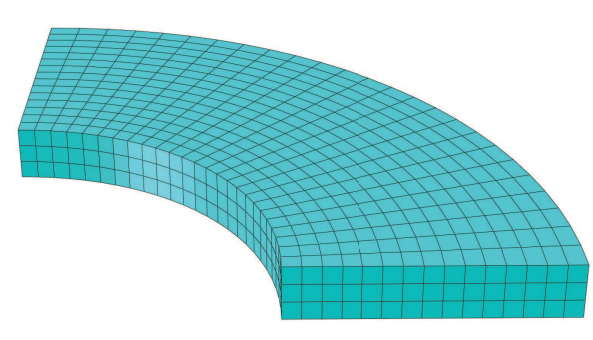

(b) Discretized cylinder

Figure I. Geometrical setup and discretisation of a quarter cylinder.

Table I. Various material constants used in the computations.

\begin{tabular}{lllll}
\hline$g_{0}[\mathrm{kPa}]$ & $g_{1}\left[\mathrm{kPa} /[\mathrm{kV} / \mathrm{m}]^{2}\right]$ & $c_{1}[\mathrm{~F} / \mathrm{m}]$ & $c_{2}[\mathrm{~F} / \mathrm{m}]$ & $\alpha[\mathrm{I} / \mathrm{K}]$ \\
\hline $10^{2}$ & $-1.0 \times 10^{-3}$ & $0.2 \varepsilon_{0}$ & $2 \varepsilon_{0}$ & $2 \times 10^{-6}$ \\
\hline
\end{tabular}

coefficient $\alpha$ and the bulk modulus $\kappa$. As has been proposed in [53], we approximate the shear modulus by $\mu\left(I_{4}\right)=g_{0}+g_{1} I_{4}$, with $g_{0}$ being the zero field shear modulus and $g_{1}<0$ characterising a softening effect of the electric field on the material. The contribution of the energy stored in the electric field represented by the term $E(\boldsymbol{F}, \mathbb{E})$ in the energy function will be neglected due to its small value [34] compared to the other energy contributions within the body.

\section{I. Inflation and extension of a cylindrical tube}

We consider the quasi-static finite strain thermo-electro-elastic expansion and inflation of a thick-walled cylindrical tube as depicted in Figure 1. This example setup is widely used in the literature, for example in [27, 29, 53-56]. Due to the symmetry of the problem the geometry is reduced to a quarter of a cylinder with an initial internal radius $A_{i}=10 \mathrm{~mm}$ and an initial external radius $A_{e}=20 \mathrm{~mm}$. In the deformed configuration these radii are denoted by $a_{i}$ and $a_{e}$.

For the following example an axial stretch is prevented by prescribing displacement boundary conditions $u_{z}=0$ on the top and bottom surfaces of the cylinder. Furthermore these surfaces are considered to be insulated so that the heat flux $\boldsymbol{q}=\mathbf{0}$, and in order to generate an axial electric field a potential difference $\Delta \phi$ can be prescribed which induces the electric field $\mathbb{E}$. In [27] and [53] this configuration is chosen as it allows for an analytical solution. Even though the axial application of an electric field for a cylindrical test subject is difficult to achieve in a real-life experiment, we chose to adapt this setup in order to validate the numerical results with the ones from the analytical calculations presented in [27]. On the internal and external radial surfaces of the cylinder a temperature field is imposed. To achieve an inflation of the tube the displacement on the internal surface is prescribed. We introduce the value $\lambda_{i}$, which is the ratio of the deformed internal radius to the initial internal radius, in order to characterise the amount of inflation.

The behaviour of the tube can be characterised by the resulting pressure on the internal surface. This is derived in [27] as

$$
p_{i}=\int_{a_{i}}^{a_{e}} \frac{1}{r}\left[\sigma_{\phi \phi}^{\mathrm{tot}}(r)-\sigma_{r r}^{\mathrm{tot}}(r)\right] \mathrm{d} r
$$

where $\sigma_{\phi \phi}^{\text {tot }}$ and $\sigma_{r r}^{\text {tot }}$ are the circumferential and radial contributions of the total stress. In the numerical calculations this integral is evaluated numerically using Gaussian quadrature. The material parameters used in the following calculations, as listed in Table 1, are identical to those used in [27] in order to best replicate the analytical results presented there. 


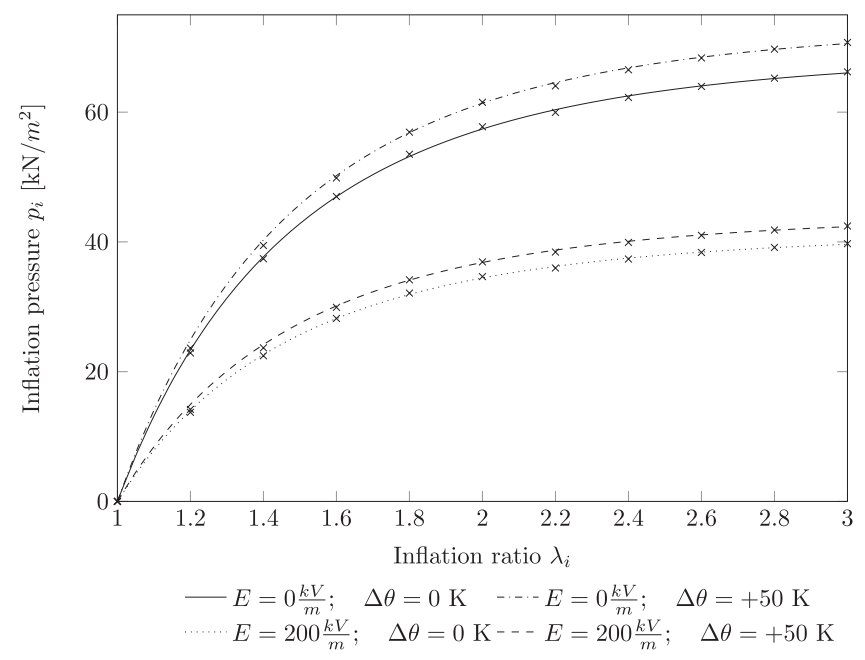

Figure 2. Resulting pressure on the internal surface of the cylinder for selected values of an axial electric field $E$ and a radial temperature difference $\Delta \Theta$.

In [27] the material was assumed to be incompressible at constant temperature. In accordance with [35], for the numerical calculations a Poisson ratio of 0.499 is selected in order to achieve an electro-mechanical material behaviour that is nearly incompressible. Linear shape functions were used in the computation without the occurrence of volume-locking due to the simple geometric setup of the problem presented here.

In order to validate the finite-element implementation, the numerically computed results are compared to the analytical results presented in [27]. It should be noted that all simplifying assumptions made in the computation of the analytical results are also applied in the numerical calculation. The structural thermo-mechanical and thermo-electric heating/cooling effects in equation (33) are therefore neglected.

Figure 2 shows both the analytical solutions as lines and the numerical results using additional marks. As expected, the behaviour of the material is sensitive both to thermal and to electric loading. The application of an electric field leads to a decrease in the pressure as the material softens due to the influence of the material parameter $g_{1}$. Conversely, the pressure increases when a radial temperature gradient is introduced which is in accordance with the results found in [57]. Generally the numerical and analytical results show very good agreement with only small discrepancies that are due to the finite-element discretisation and the numerical evaluation of the integral in the definition of the pressure given by equation (49).

Using the validated numerical implementation above, some of the simplifications made in the derivation of the analytical results can now be discarded. Thus the thermo-mechanical and the thermo-electric effects that were neglected before are reinstated. As the considered material is nearly incompressible the thermo-mechanical effect is significantly smaller than the electro-thermal effect, and it will therefore not be analysed specifically in this context.

Figure 3(a) shows the pressure on the inside of the tube $\left(R=A_{i}\right)$ when the electric field is applied by linearly increasing the electric potential on the top and bottom surfaces of the cylinder from zero to $\Delta \varphi^{\max }$ over a ramp time $t_{r}$ at fixed deformation. Thus each data point in Figure 3(a) is captured at a constant deformation and respective ramp time for the electric field. In contrast to the first example the temperature on the external surface of the cylinder is not fixed; rather, an insulating boundary condition is applied.

It is observed in Figure 3(a) that for the given material parameters and loading conditions stress generation is still dominated by the mechanical deformation. The pressure decreases as the timespan for the application of the electric field is reduced. As presented in the previous example the decrease in pressure is a result of the decreased temperature of the cylinder which in turn is caused by the electric cooling effect. The temperature change in this example is negative due to the combination of parameters, as the effect is dominated by the negative material parameter $g_{1}$ when compared to the positive parameters $c_{1}$ and $c_{2}$. This temperature decrease becomes larger for shorter ramp times of the electric field and therefore the change in the pressure becomes more distinct. Figure 3(b) shows the temperature difference on the external surface of the cylinder at a fixed deformation $\lambda_{i}$ for varying ramp times of the electric field. For ramp times close to zero the temperature increase 


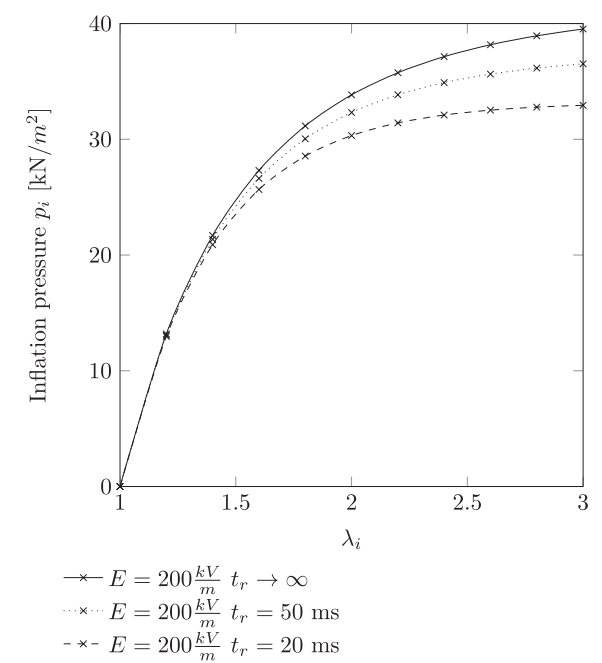

(a) Resulting pressure on the internal surface of the cylinder

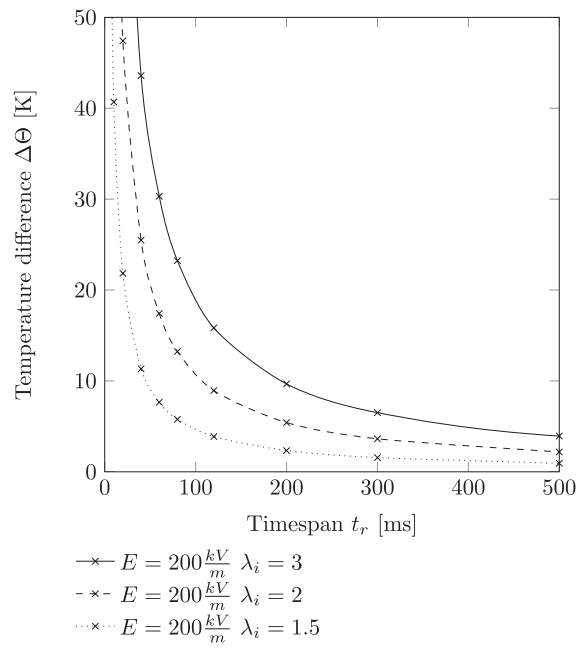

(b) Temperature difference between internal and external surface

Figure 3. Resulting pressure on the internal surface and temperature on the external surface.

Table 2. Various material constants used in the validation.

\begin{tabular}{lllll}
\hline$g_{0}[\mathrm{kPa}]$ & $g_{1}\left[\mathrm{kPa} /[\mathrm{kV} / \mathrm{m}]^{2}\right]$ & $c_{1}[\mathrm{~F} / \mathrm{m}]$ & $c_{2}[\mathrm{~F} / \mathrm{m}]$ & $\alpha[\mathrm{I} / \mathrm{K}]$ \\
\hline $10^{2}$ & 0 & $0.2 \varepsilon_{0}$ & $2 \times 10^{2} \varepsilon_{0}$ & $20 \times 10^{-6}$ \\
\hline
\end{tabular}

is significantly larger, whereas for longer ramp times the change nearly vanishes as the time derivative in equation (33) approaches zero. Furthermore, in Figure 3(b) it can also be observed that the electro-thermal heating effect is also deformation-dependent. For a larger inflation of the cylinder the temperature change becomes more distinct as the electric displacement $\mathbb{D}$ in equation (33) is deformation-sensitive.

\subsection{Deformation of a plate under electric potential loading}

With the previous example we were able to validate the derived thermo-electro-mechanical system with a boundary value problem consisting of a very simple geometric setup. As a second example we will now introduce a more complex geometry that has also been treated extensively in the literature (see $[20,34])$. In this section, the response of a three-dimensional plate (Figure 4) subjected to electric potential loading is examined. Only numerical results obtained by a finite-element simulation are presented, as there is no reference solution to this problem due to its geometric complexity. We consider a plate with a width of $40 \mathrm{~mm}$, a length of $120 \mathrm{~mm}$ and a thickness of $5 \mathrm{~mm}$. At the centre of the plate a hole is cut out with a diameter of $20 \mathrm{~mm}$. The plate is assumed to be of a neo-Hookean-type material similar to that in the previous example, but with slightly different material parameters, given in Table 2. While the zero field shear modulus has the same value as in the previous example, we will not consider a softening effect of the electric field on the material, but rather concentrate on the deformation of the plate due to the applied potential difference. Therefore, the softening parameter $g_{1}$ is set to zero and the coupling parameter $c_{2}$ is increased by a factor of $10^{3}$ in order to induce a significant material response. Since the electro-mechanical subproblem is considered to be almost incompressible we select a Poisson ratio of $v=0.495$.

By imposing an electric potential of $\varphi^{+}$and $\varphi^{-}$at the ends of the plate a potential difference $\Delta \varphi=\varphi^{+}-\varphi^{-}$ is prescribed. Additionally, at the internal surface of the hole the reference temperature $\Theta_{0}=293 \mathrm{~K}$ is imposed whereas the rest of the surfaces are assumed to be insulated.

For the numerical simulation, only a quarter of the plate is modelled with a mesh consisting of 500 linear eight-node hexahedral elements. At the resulting cut surfaces symmetry boundary conditions are imposed. Furthermore, the displacement in the $z$-direction is set to zero on the top and bottom faces of the plate.

This setup results in a two-dimensional motion of the plate. The resulting deformation and temperature change are depicted in Figure 5 for the three different electric potential loading cases corresponding to $\Delta \varphi=$ 


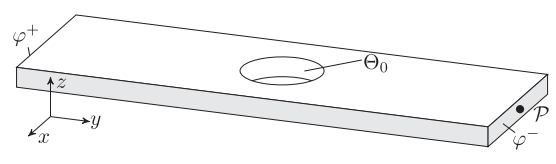

(a) Geometric setup

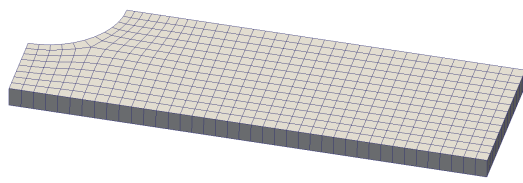

(b) Finite element mesh

Figure 4. Geometric setup of the plate and finite-element mesh of a quarter of the plate.

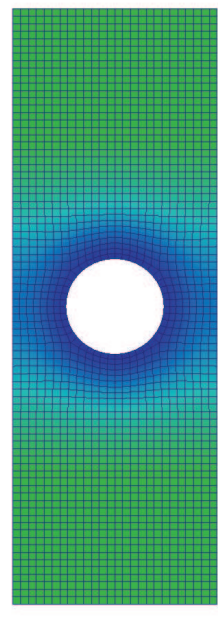

(a) Potential difference (b) Potential difference $50 \mathrm{KV}$

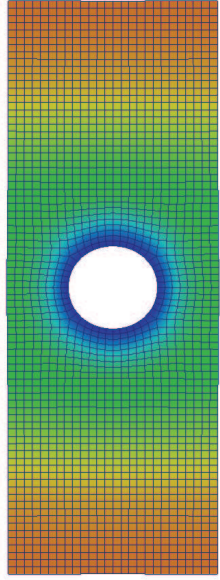

$100 \mathrm{KV}$

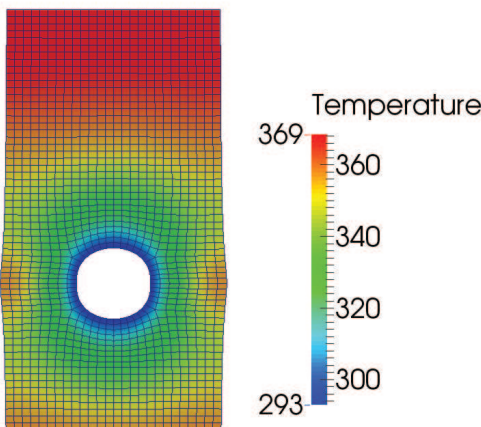

(c) Potential difference $150 \mathrm{KV}$

Figure 5. Resulting temperature plotted on the deformed plate with the initial grid at $t_{r}=I \mathrm{~s}$.

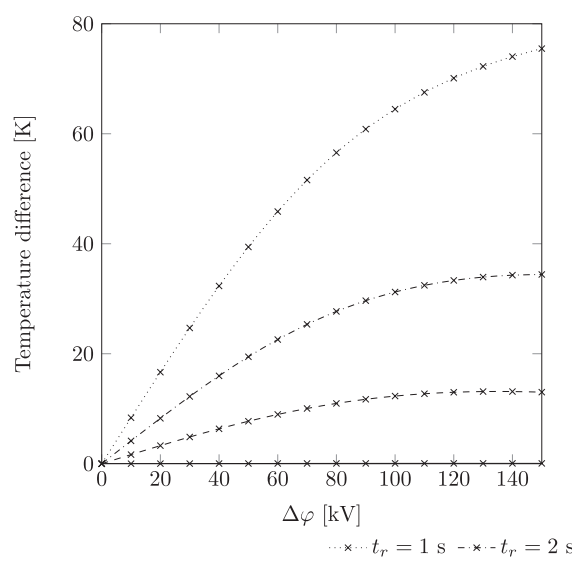

(a) Temperature on bottom surface

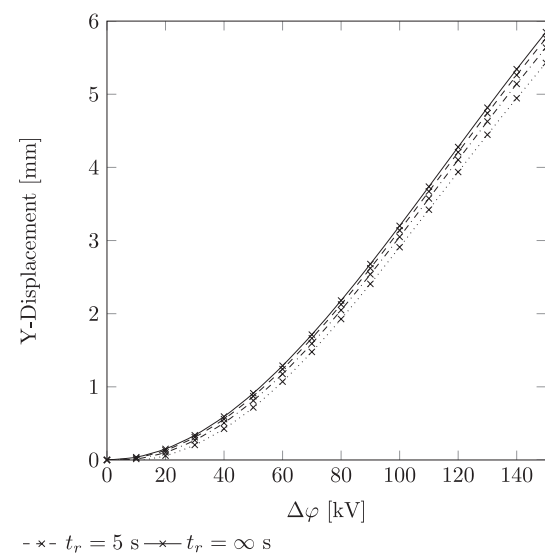

(b) Displacement on bottom surface

Figure 6. Resulting temperatures and displacements on bottom surface at different $t_{r}$.

50,100 and $150 \mathrm{kV}$. In this case it is assumed that the maximum potential difference is reached after a ramp-up time of $1.0 \mathrm{~s}$.

Figure 6 shows the displacement and the temperature change of a representative point $\mathcal{P}$ at one end of the plate, marked by a dot in Figure 4, as a function of the applied electric potential for different ramp-up times $t_{r}$, the time until the maximum electric potential difference is reached.

It can be observed in Figure 6(a) that for long ramp-up times of the electric field there is no significant temperature change at the reference point. With a decreased ramp-up time of the electric field a nonlinear increase in the temperature occurs. When compared to the first example, it is apparent that here the temperature change is positive which is due to the chosen material parameters. As we are not considering a softening effect of the electric field on the material, the parameter $g_{1}$ is set to zero and therefore the heating effect is dominated 


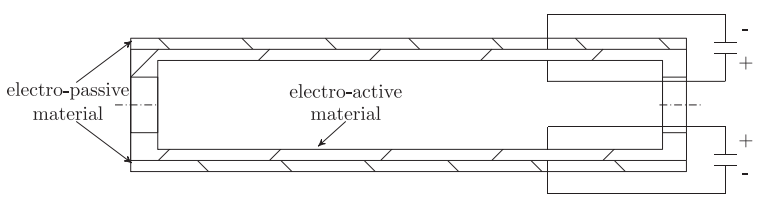

(a) Geometric setup

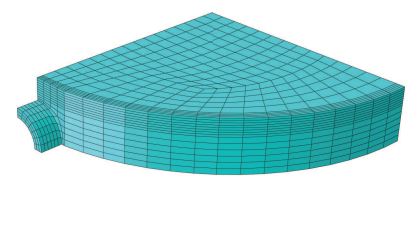

(b) Finite element mesh

Figure 7. (a) Geometric setup of the pump and (b) finite-element mesh of one-eighth of the pump.

by the positive material parameters $c_{1}$ and $c_{2}$ which leads to a temperature increase. This in turn leads to an expansion of the material that acts against the contractions of the plate due to the electric field. Resulting from this is a reduced displacement of the reference point, which is depicted in Figure 6(b). It should be noted that this temperature change of almost $100 \mathrm{~K}$ at a ramp-up time of $3 \mathrm{~s}$ is artificially large due to the high value of the coupling parameter $c_{2}$ that was chosen in order to induce a significant deformation of the material. Nevertheless, the results show that the influence of the temperature on the material behaviour must not be neglected.

\subsection{Temperature influence on a microfluidic pumping device}

In this final example we will investigate a geometry that is more application-oriented, namely a cylindrical microfluidic pumping device, sketched in Figure 7(a), which is based on the concept presented in [58]. Such a device might, for example, be used as an implant for the controlled micro-injection of drugs [59]. The pumping function is achieved by a unimorph diaphragm actuator which consists of an electro-active layer sandwiched between two compliant electrodes that is covered on one side with a thin film of electro-passive material. Upon stimulation by an electric field in the thickness direction the actuator reacts by bulging in the direction of the active layer.

The geometry of the idealised pump consists of a cylinder of electro-active material with a thickness of $40 \mu \mathrm{m}$ for the curved walls and $20 \mu \mathrm{m}$ for the top and bottom, an internal radius of $460 \mu \mathrm{m}$ and an internal height of $160 \mu \mathrm{m}$. The top and the bottom surfaces are sandwiched between two compliant electrodes. Additionally, the cylinder has two holes of radius $50 \mu \mathrm{m}$ for fluid inflow and outflow on opposing sides. Over the entire top and bottom surfaces of the cylinder we add a layer of electro-passive material with a thickness of $20 \mu \mathrm{m}$. Due to the geometric and loading symmetry we model only one-eighth of the pump including a part of the inflow/outflow channels for the finite-element solution of the problem, as shown in Figure 7(b). The thermo-mechanical system is solved in 20 time steps over which the induced electric field is linearly increased. As the geometry and the resulting deformation are more complex than in the previous examples, we use an initial coarse mesh with approximately 3000 hexahedral elements to which we apply adaptive mesh refinement to increase the accuracy of the solution. For this an h-refinement method is employed after the first and tenth steps, and a Kelly error estimator is used to determine regions in which the solution has a large gradient. This leads to a final mesh consisting of 8000 elements with finer discretisation at the locations of electric field singularities. We prescribe a potential difference over the thickness of the electro-active layer. For the mechanical boundary conditions we use symmetry boundary conditions on the cut surfaces of the model. Additionally, we constrain the deformation of the end face of the inflow/outflow channel as we assume them to be rigidly connected. On the internal surface we prescribe a temperature that is equal to that of the pumping fluid, whereas on the entire external surface we prescribe a temperature equal to that of the surrounding environment.

We primarily focus on the case where the fluid temperature changes by $\pm 50 \mathrm{~K}$ and the temperature of the surrounding environment remains at a reference temperature of $293 \mathrm{~K}$, thus resulting in a temperature difference $\Delta \Theta$ between the internal and external surfaces. As additional temperature configurations we also investigate two other cases. First we reverse the thermal configuration so that the fluid temperature remains at the reference temperature of $293 \mathrm{~K}$ and the temperature of the environment is altered. Second, the overall temperature of the system is altered by $50 \mathrm{~K}$ so that on the internal and external surfaces the same temperature is prescribed. Therefore there is no temperature gradient in this configuration. As no experimental data is available on the heat transfer between the pump and the fluid or the surroundings, we focus solely on the influence of the fluid temperature and neglect the thermo-mechanical and thermo-electric heating effects as secondary heat sources for the scope of this example. The electro-active layers are assumed to have the same properties as in the previous example whereas the electro-passive body of the pump consists of a decoupled neo-Hookean material 

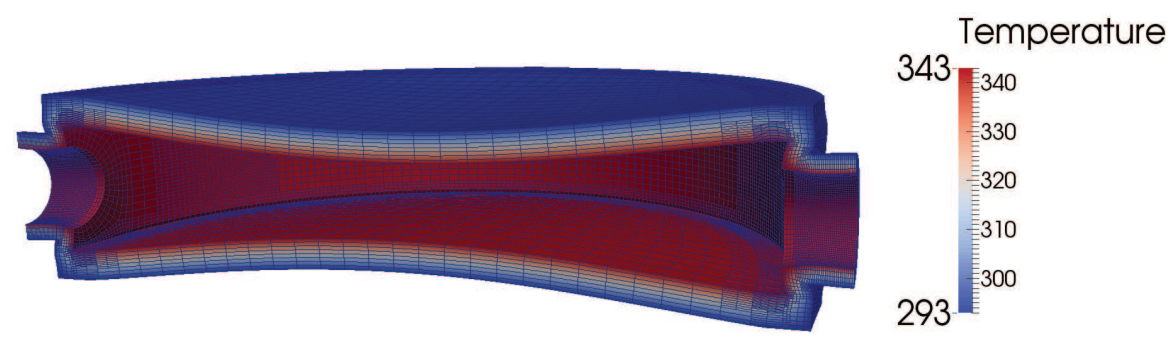

Figure 8. Deformation of the pump for a fluid with the temperature $343 \mathrm{~K}$. The colour map refers to the temperature distribution throughout the model.

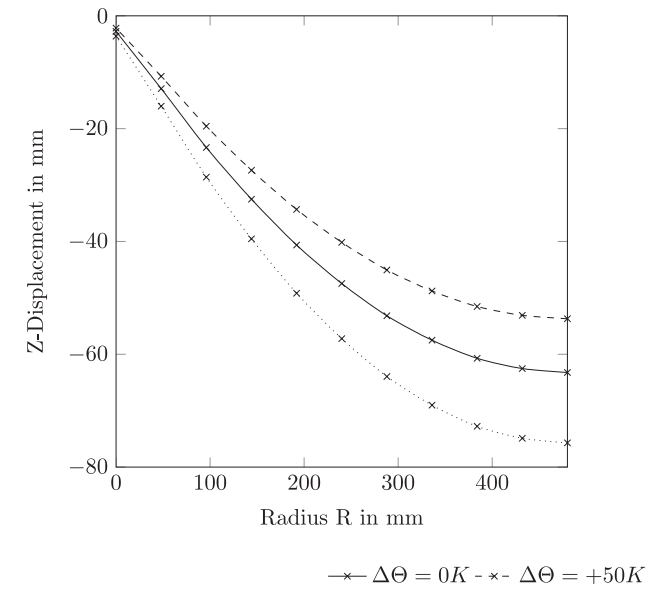

(a) Variation over the undeformed internal radius of the pump

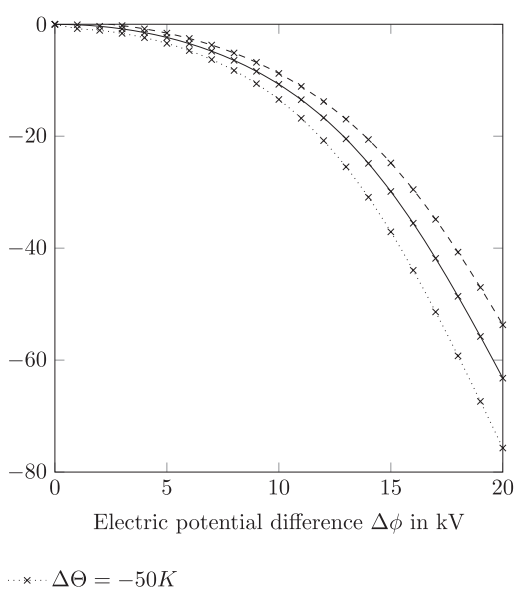

(b) Influence of the electric potential difference

Figure 9. Deformation of the pump in the axial direction for various temperature differences $\Delta \Theta$ between the internal and external surfaces.

with the same mechanical properties as the active material. We apply a maximum voltage of $20 \mathrm{kV}$ to both actuators. Figure 8 depicts half of the deformed pump for a fluid with a temperature of $343 \mathrm{~K}$.

The deformed plot of the model shows that the wall of the pump flexes as the top and bottom surfaces contract inwards, thereby decreasing the internal volume of the pump. This motion is the result of the arrangement of the active and the passive material layers. When the electric field is applied in the thickness direction of the actuator, the active layer contracts in the direction of the electric field and therefore expands in the transverse direction. The deformation of the active layer leads to a compression of the passive thin film resulting in the inwards bulging of the top and bottom surfaces of the pump. On the inside of the pump, as well as on the internal surfaces of the inflow and outflow channels, the temperature is equal to the fluid temperature whereas the external surfaces are kept at the reference temperature of $293 \mathrm{~K}$, leading to a significant temperature gradient through the thickness of the material. The left plot in Figure 9 presents the displacement in the axial direction over the undeformed internal radius of the pump when the temperature on the external surface of the device is kept at $293 \mathrm{~K}$ while changing the temperature of the pumping fluid from $293 \mathrm{~K}$ by $\pm 50 \mathrm{~K}$.

It is clear that the temperature has a significant influence on the deformation and thus on the volume stream that the pump provides. When the temperature on the internal surface of the pump is increased the resulting displacement of the actuators decreases, whereas the displacement increases with decreasing temperature. The right plot in Figure 9 depicts the deformation in the axial direction of a point in the centre of the internal top surface of the pump, as governed by the applied electric field. It exhibits a nonlinear dependence of the deformation on the electric potential difference and clearly illustrates that the thermal field affects the deflection of the diaphragm throughout the range of the induced electric fields.

When we alter the thermal boundary conditions of this example, the way the temperature influences the functionality of the pump can be analysed further. We calculated the results for all of the following temperature configurations but do not present them explicitly. Instead these results are used for comparison with the ones of 


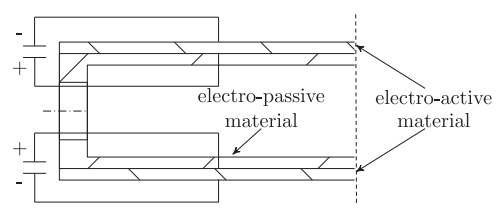

(a) Geometric setup

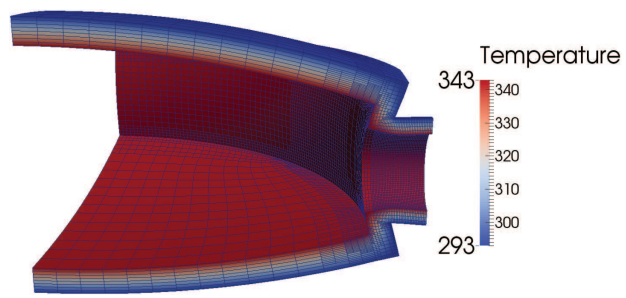

(b) Deformation of the reservoir

Figure 10. (a) Geometric setup and (b) deformed plot of the reservoir. The colour map refers to the temperature distribution throughout the model.

the initial experiments to illustrate the effect of the thermal configuration. We reversed the previous temperature setup by keeping the temperature on the internal surface fixed at the reference temperature and changing the temperature on the external surface by $50 \mathrm{~K}$. Comparing with the previous configuration, the influence of the temperature was reversed in this case, as the displacement increased when the external surface was heated up but decreased when the outer surface of the pump was cooled down. From this observation we can conclude that the direction of the temperature gradient is the critical factor for the deformation of the pump, not the absolute value of the temperature.

As a final thermal setup we changed the overall temperature of the experiment, so both the internal and external surfaces of the pump were at equal temperatures and heated up or cooled down by $50 \mathrm{~K}$. In this configuration it could be observed that in the absence of a temperature gradient the functionality of the pump remains consistent when the temperature is changed. From this we can conclude that, for the chosen material properties, the influence of the temperature on the deformation of the pump is only relevant when a temperature gradient exists between the inside and outside surfaces of the pump. One has to keep in mind that in this contribution the material parameters are assumed to be temperature-independent. An overall change in the temperature may be expected to have an influence on the deformation of the pump once a temperature dependency of the material parameters is introduced. As suggested in [58] the pump can be combined with an active reservoir that expands in order to receive the fluid. We can use our previous model to simulate this supporting device by switching the order of the material assembly.

As depicted in Figure 10(a) the cylindrical body now consists of an electro-passive material to which we add an electro-active layer sandwiched between compliant electrodes on the top and bottom surfaces. The resulting deformation of the model is reversed when compared to the one of the pump. When an electric field is applied, the reservoir expands in order to support the function of the pump. Figure 10(b) depicts a quarter of the deformed reservoir for a fluid with the temperature of $343 \mathrm{~K}$. The deformed plot of the FEM-model shows that the wall of the reservoir deflects as the top and bottom surfaces expand outwards, thereby increasing the internal volume of the reservoir. This reversed motion is the result of the altered arrangement of the active and the passive material layers. Upon electric stimulation the active layer contracts in the direction of the electric field and therefore expands transversely. This leads to a compression of the passive thin film, which is now the internal surface of the model, resulting in the bulging in the outward direction of the top and bottom surfaces of the reservoir. Next we analyse the influence of the temperature on the behaviour of the reservoir. We keep the temperature on the external surface fixed at $293 \mathrm{~K}$ and investigate the same three cases for the temperature of the fluid. Thus the internal temperature is either kept at the reference temperature or changed by $\pm 50 \mathrm{~K}$. Figure 11 (a) depicts the displacement in the axial direction over the undeformed internal radius of the reservoir for these three temperature configurations, whereas Figure 11(b) shows the deformation in the axial direction of a point in the centre of the internal top surface of the reservoir, as governed by the applied electric field.

Both plots show that even though the electric potential applied to the actuators of the reservoir is the same as the one on the pump, the magnitude of the displacement is considerably smaller. Furthermore, the deformation of the reservoir is affected by the temperature in a fashion similar to the pump. When we increase the temperature on the internal surface we see that the deformation increases, whereas the displacement is reduced when the internal surface is cooled down. This behaviour confirms that the direction of the temperature gradient is the dominating factor in the influence of the temperature on the deformation. A positive temperature gradient in the direction of the bulging supports the deformation whereas a negative temperature gradient counteracts it. 


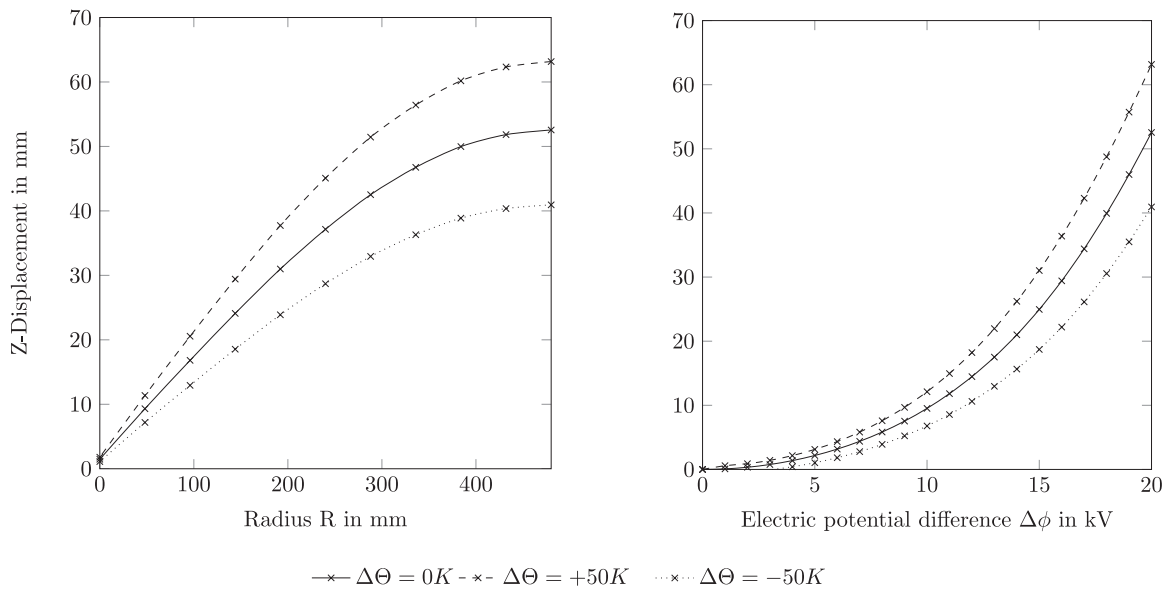

(a) Axial displacement over the internal radius of (b) Axial displacement depending on the the reservoir electric potential difference

Figure I I. Deformation of the reservoir in the axial direction for various temperature differences $\Delta \Theta$ between the internal and external surfaces.

\section{Conclusion and future work}

Despite the common perception that temperature can have a significant impact on the behaviour of rubber-like materials, the influence of thermal effects on electro-active polymers has, to the best of the authors' knowledge, been investigated in only a small number of contributions [60]. Therefore, the formulation of a thermo-electromechanically coupled modelling framework and its implementation in a simulation environment is essential in order to realistically predict experimental results. In this contribution, we have presented a thermo-electromechanically coupled constitutive framework that obeys the relevant laws of thermodynamics and its solution using the finite-element method. A simplified implementation was compared to analytical results of a widely used non-homogeneous boundary value problem for validation purposes. Thereafter the numerical model was extended to include thermo-mechanical and thermo-electric heating/cooling effects. Depending on the material parameters and the loading conditions it can be shown that these thermal effects can have a significant influence on the material response. At the moment, however, essential time-dependent effects, such as the inelasticity of the underlying polymeric materials, have been ignored. The presented model therefore needs to be extended to include, for example, viscoelastic dissipative behaviour. Future work also includes the conducting of experimental studies in order to identify constitutive material parameters and to validate the model with real experimental data.

\section{Funding}

The authors acknowledge the funding received within the Deutsche Forschungsgemeinschaft (project number STE 544/52-1). J-P Pelteret and P Steinmann would like to express their sincere gratitude for the funding by the European Research Council (ERC) (advanced grant 289049 MOCOPOLY).

\section{References}

[1] Maugin, G, and Eringen, A. On the equations of the electrodynamics of deformable bodies of finite extent. J Méc 1977; 16: 101-147.

[2] Maugin, GA. A continuum theory of deformable ferrimagnetic bodies. I. Field equations. J Math Phys 1976; 17(9): 1727-1738.

[3] Maugin, GA, and Eringen, AC. Deformable magnetically saturated media. I. Field equations. J Math Phys 1972; 13 (2): $143-155$.

[4] Maugin, G. Continuum mechanics of electromagnetic solids. Amsterdam: North-Holland, 1988.

[5] Eringen, AC, and Maugin, GA. Electrodynamics of continua I: Foundations and solid media. New York, NY: Springer Science \& Business Media, 2012.

[6] Maugin, GA. Material inhomogeneities in elasticity, vol. 3. Boca Raton, FL: CRC Press, 1993.

[7] Epstein, M, and Maugin, G. Inhomogeneities, Eshelby's tensor and $J$-integral in electroelasticity. In: Hsieh, RKT (ed.) Mechanical modelling of new electromagnetic materials. Amsterdam: Elsevier, 1990, 253-258. 
[8] Epstein, M, and Maugin, G. Energy-momentum tensor and J-integral in electrodeformable bodies. Int J Appl Electromag Mater 1990; 2(2): 141-145.

[9] O'Halloran, A, O'Malley, F and McHugh, P. A review on dielectric elastomer actuators, technology, applications, and challenges. J Appl Phys 2008; 104(7): 9.

[10] Pelrine, R, Kornbluh, R, and Kofod, G. High-strain actuator materials based on dielectric elastomers. Adv Mater 2000; 12(16): 1223-1225.

[11] Bar-Cohen, Y. Electroactive polymers: Current capabilities and challenges. In: SPIE's 9th Annual International Symposium on Smart Structures and Materials, 1-7.

[12] Bar-Cohen, Y. Electroactive polymer (EAP) actuators as artificial muscles: Reality, potential, and challenges, vol. 136, Bellingham, WA: SPIE Press, 2004.

[13] Vertechy, R, Fontana, M, Rosati Papini, GP, et al. In-tank tests of a dielectric elastomer generator for wave energy harvesting. In: SPIE Smart Structures and Materials + Nondestructive Evaluation and Health Monitoring, 90561G-90561G, Bellingham, WA: International Society for Optics and Photonics, 2014.

[14] Böse, H, and Fuß, E. Novel dielectric elastomer sensors for compression load detection. In: SPIE Smart Structures and Materials + Nondestructive Evaluation and Health Monitoring, 905,614-905,614.

[15] Koh, SJA, Keplinger, C, Li, T, et al. Dielectric elastomer generators: How much energy can be converted? IEEE/ASME Trans Mechatron 2011; 16(1): 33-41.

[16] Pelrine, R, Kornbluh, R, Pei, Q, et al. High-speed electrically actuated elastomers with strain greater than 100\%. Sci 2000; 287(5454): 836-839.

[17] Brochu, P, and Pei, Q. Advances in dielectric elastomers for actuators and artificial muscles. Macromolec Rapid Commun 2010; 31(1): 10-36.

[18] Bustamante, R. Transversely isotropic non-linear electro-active elastomers. Acta Mech 2009; 206(3): $237-259$.

[19] Ask, A, Menzel, A, and Ristinmaa, M. Phenomenological modeling of viscous electrostrictive polymers. Int J Non-Lin Mech 2012; 47(2): 156-165.

[20] Ask, A, Menzel, A, and Ristinmaa, M. Electrostriction in electro-viscoelastic polymers. Mech Mater 2012; 50: 9-21.

[21] Johlitz, M, Steeb, H, Diebels, S, et al. Experimental and theoretical investigation of nonlinear viscoelastic polyurethane systems. J Mater Sci 2007; 42(23): 9894.

[22] Diaconu, I, Dorohoi, DO, and Ciobanu, C. Electromechanical response of polyurethane films with different thickness. Rom J Phys 2006; 53(1-2): 91-97.

[23] Büschel, A, Klinkel, S, and Wagner, W. Dielectric elastomers-numerical modeling of nonlinear visco-electroelasticity. Int $J$ Numer Meth Eng 2013; 93(8): 834-856.

[24] Saxena, P, Hossain, M, and Steinmann, P. A theory of finite deformation magneto-viscoelasticity. Int J Solid Struct 2013; 50(24): 3886-3897.

[25] Saxena, P, Vu, DK, and Steinmann, P. On rate-dependent dissipation effects in electro-elasticity. Int J Non-Lin Mech 2014; 62: $1-11$.

[26] Park, HS, Suo, Z, Zhou, J, et al. A dynamic finite element method for inhomogeneous deformation and electromechanical instability of dielectric elastomer transducers. Int J Solid Struct 2012; 49(15): 2187-2194.

[27] Mehnert, M, Hossain, M, and Steinmann, P. On nonlinear thermo-electro-elasticity. Proc R Soc A 2016; 472(2190): 20160170.

[28] Felippa, CA, Park, K, and Farhat, C. Partitioned analysis of coupled mechanical systems. Comput Meth Appl Mech Eng 2001; 190(24): 3247-3270.

[29] Erbts, P, and Düster, A. Accelerated staggered coupling schemes for problems of thermoelasticity at finite strains. Comput Math Appl 2012; 64(8): 2408-2430.

[30] Erbts, P, Hartmann, S, and Düster, A. A partitioned solution approach for electro-thermo-mechanical problems. Arch Appl Mech 2015; 85(8): 1075-1101.

[31] Voltairas, P, Fotiadis, D, and Massalas, C. A theoretical study of the hyperelasticity of electro-gels. Proc R Soc London A: Math Phys Eng Sci 2003; 459: 2121-2130.

[32] Griffiths, DJ. Introduction to electrodynamics, 2nd ed. Englewood Cliffs, NJ: Prentice Hall, 1989.

[33] Dorfmann, A, and Ogden, R. Nonlinear electroelasticity. Acta Mech 2005; 174(3-4): 167-183.

[34] Vu, D, Steinmann, P, and Possart, G. Numerical modelling of non-linear electroelasticity. Int J Numer Meth Eng 2007; 70(6): 685-704.

[35] Vogel, F. On the modeling and computation of electro-and magneto-active polymers (Dissertation). Lehrstuhl für Technische Mechanik, Universität Erlangen-Nürnberg, 2015.

[36] Bustamante, R. A variational formulation for a boundary value problem considering an electro-sensitive elastomer interacting with two bodies. Mech Res Commun 2009; 36(7): 791-795.

[37] Vogel, F, Göktepe, S, Steinmann, P, et al. Modeling and simulation of viscous electro-active polymers. Eur J Mech A/Solid 2014; 48: 112-128.

[38] Steinmann, P. Computational nonlinear electro-elasticity - getting started. In: Ogden, RW and Steigmann, DJ (eds) Mechanics and electrodynamics of magneto- and electro-elastic materials. New York, NY: Springer, 2011, 181-230.

[39] Holzapfel, GA. Nonlinear solid mechanics, vol. 24. Chichester, UK: Wiley, 2000. 
[40] Miehe, C. Entropic thermoelasticity at finite strains. Aspects of the formulation and numerical implementation. Comput Meth Appl Mech Eng 1995; 120(3-4): 243-269.

[41] Vu, D. A study on nonlinear electro-elastostatics: Theory and numerical simulation (Habilitation). Friedrich-Alexander University of Erlangen-Nürnberg: Erlangen, Bayern, Germany, 2014.

[42] Coleman, BD, and Noll, W. The thermodynamics of elastic materials with heat conduction and viscosity. Arch Rat Mech Anal 1963; 13(1): 167-178.

[43] Dorfmann, A, and Ogden, R. Magnetoelastic modelling of elastomers. Eur J Mech A/Solid 2003; 22(4): $497-507$.

[44] Dorfmann, A, and Ogden, R. Nonlinear magnetoelastic deformations of elastomers. Acta Mech 2004; 167(1): 13-28.

[45] Ibrahimbegovic, A, Kožar, I, Marović, P, et al. Multiscale in time and stability analysis of operator split solution procedures applied to thermomechanical problems. Eng Comput 2009; 26(1/2): 205-223.

[46] Bangerth, W, Hartmann, R, and Kanschat, G. Deal. II - A general-purpose object-oriented finite element library. ACM Trans Math Softw (TOMS) 2007; 33(4): 24.

[47] Bangerth, W, Heister, T, Heltai, L, et al. The deal.II library, version 8.2. Arch Numer Softw 2015; 3(100): 1-8.

[48] Heroux, MA, Bartlett, RA, Howle, VE, et al. An overview of the Trilinos Project. ACM Trans Math Softw 2005; 31 (3): $397-423$.

[49] Heroux, MA, and Willenbring, JM. Trilinos user's guide, 2003. https://trilinos.org/oldsite/TrilinosUserGuide.pdf

[50] Burstedde, C, Wilcox, LC, and Ghattas, O. p4est: Scalable algorithms for parallel adaptive mesh refinement on forests of octrees. SIAM J Sci Comput 2011; 33(3): 1103-1133.

[51] Pelteret, JP, Davydov, D, McBride, A, et al. Computational electro-elasticity and magneto-elasticity for quasi-incompressible media immersed in free space. Int J Numer Meth Eng 2016; 108(11): 1307-1342.

[52] Eringen, AC. On the foundations of electroelastostatics. Int J Eng Sci 1963; 1(1): 127-153.

[53] Bustamante, R. Transversely isotropic nonlinear magneto-active elastomers. Acta Mech 2010; 210(3-4): $183-214$.

[54] Armero, F, and Simo, J. A new unconditionally stable fractional step method for non-linear coupled thermomechanical problems. Int J Numer Meth Eng 1992; 35(4): 737-766.

[55] Saxena, P, Pelteret, JP, and Steinmann, P. Modelling of iron-filled magneto-active polymers with a dispersed chain-like microstructure. Eur J Mech A/Solid 2015; 50: 132-151.

[56] Dorfmann, A, and Ogden, R. Nonlinear electroelastic deformations. J Elast 2006; 82(2): 99-127.

[57] Treloar, L, and Montgomery, D. The physics of rubber elasticity. Phys Tod 1959; 12(2): 32-34.

[58] Piyasena, ME, Newby, R, Miller, TJ, et al. Electroosmotically driven microfluidic actuators. Sens Act B: Chem 2009; 141(1): 263-269.

[59] Yan, B, Li, B, Kunecke, F, et al. Polypyrrole-based implantable electroactive pump for controlled drug microinjection. ACS Appl Mater \& Interf 2015; 7(27): 14,563-14,568.

[60] Elahinia, M, Vertechy, R, Berselli, G, et al. Continuum thermo-electro-mechanical model for electrostrictive elastomers. J Int Mater Syst Struct 2013; 24(6): 761-778. 